\title{
Mimickers and tumours in the lower urinary tract: Do we need more efficient vigilance?
}

\author{
Peter D. Metcalfe, MD, MSc, FRCSC; ${ }^{*}$ Alex Bascom, MD, BSc, ${ }^{*}$ Consolato Sergi, MSc, MD, PhD ${ }^{\dagger}$
}

*Department of Surgery, University of Alberta, Edmonton, AB; ${ }^{\dagger}$ Department of Laboratory Medicine and Pathology, University of Alberta, Edmonton, AB

Cite as: Can Urol Assoc J 2013;7(5-6):421-5. http://dx.doi.org/10.5489/cuaj.1386 Published online June 12, 2013.

\section{Abstract}

Lower urinary tract symptoms are common in pediatric urology, but an atypical presentation may portend significant pathophysiology. We present 5 cases of young males with a sudden change in urinary symptoms, which resulted in the discovery of a variety of benign bladder tumours. We present the clinical, radiologic and histologic findings of these cases emphasizing on mimickers in clinics. Although rare tumours, these may be increasing in frequency and pediatric care may further be enhanced by the development of multinational registry.

\section{Introduction}

When pediatric patients present with a sudden onset of lower urinary tract symptoms, it is important to include some of the rare diagnoses in the differential. This report focus on 5 peculiar cases in boys: a 4-year-old with a fibrovascular polypoid of the verumontanum, a papilloma in a newborn, a hemangioma of the prostate in a 4-year-old, and 2 teenagers with papillary urothelial neoplasms of low malignant potential (PUNLMP).

Pediatric tumours of the lower urinary tract are rare, ${ }^{1-3}$ but may portend significant consequences. ${ }^{3}$ According to a recent review by Alanee and Shukla, the incidence of bladder tumours in the pediatric population has increased. ${ }^{4}$ Although most cases from the Surveillance Epidemiology and End Results (SEER) database had a favourable outcome, aggressive malignancies do occur. ${ }^{2}$ We have identified a small series of bladder tumours over a short period, which merit discussion about current vigilance. All 5 children originally presented with common symptoms, but careful historytaking resulted in detecting subtle differences. Liberal use of imaging was also integral to the early diagnosis.
Although bladder tumours in children are rare, they are of significant concern. Therefore, a multinational registry may be necessary to improve our knowledge regarding etiology, treatment options and surveillance.

\section{Case 1}

A 4-year-old boy was referred to urology for investigation of increased frequency, recurrent urinary tract infections (UTIs) and lower abdominal pain. An ultrasound demonstrated a $0.5 \times 0.8-\mathrm{cm}$ polypoid mass within the bladder adjacent to the bladder neck (Fig. 1). At cystoscopy, a benign appearing polyp was found just proximal to the verumontanum. Otherwise urethra, ureteric orifices and bladder were normal. A 9-French resectoscope was inserted and hook cautery was used to resect the tumour from its base.

Microscopy demonstrated a benign and rare variant of a fibrovascular polyp of the verumontanum (Fig. 2). Transitional cells lined the epithelium, covering thick, dense stroma with blood vessels, nerve fibers and sections of ducts. No significant cellular atypical or cytological abnormality was observed. On the surface, there was transitional cell epithelium, covering thick, dense stroma with blood vessels, nerve fibers and a couple of sections of ducts with secrete in the lumen. No significant cellular atypia was observed. An internal evaluation for quality assurance (second opinion) confirmed the diagnosis.

Postoperative 3- and 12-month ultrasounds have demonstrated no further evidence of tumour. The patient was experiencing less penile pain and bladder spasms immediately postoperatively, and has remained symptom free.

\section{Case 2}

A 3-day-old male underwent an ultrasound as routine follow-up for mild unilateral hydronephrosis. Although the hydronephrosis resolved, a $0.3 \times 0.3-\mathrm{cm}$ papillary mass was detected at the trigone (Fig. 3). The baby was otherwise 


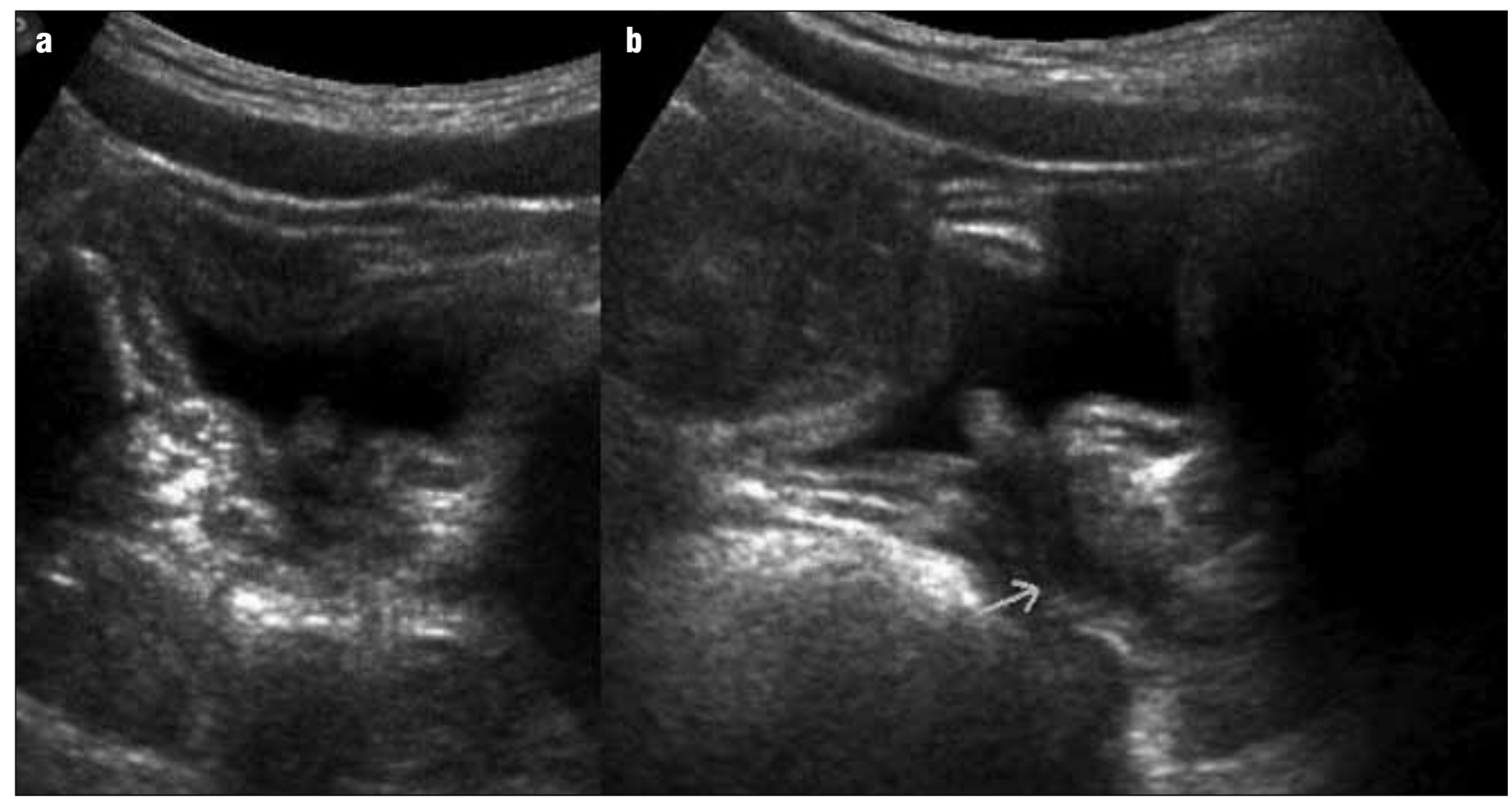

Fig. 1. An ultrasound of the bladder neck demonstrating a $0.5 \times 0.8-\mathrm{cm}$ polypoid lesion protruding into the bladder; $A$ : transverse, $B$ : sagittal views.

healthy, and follow-up ultrasound 1 and 3 months later did not demonstrate any significant growth. Endoscopic resection was performed at the age of 3 months without any difficulty. Pathology was consistent with a papilloma, with no malignant features. Follow-up ultrasound at 3 months was negative for a recurrence.

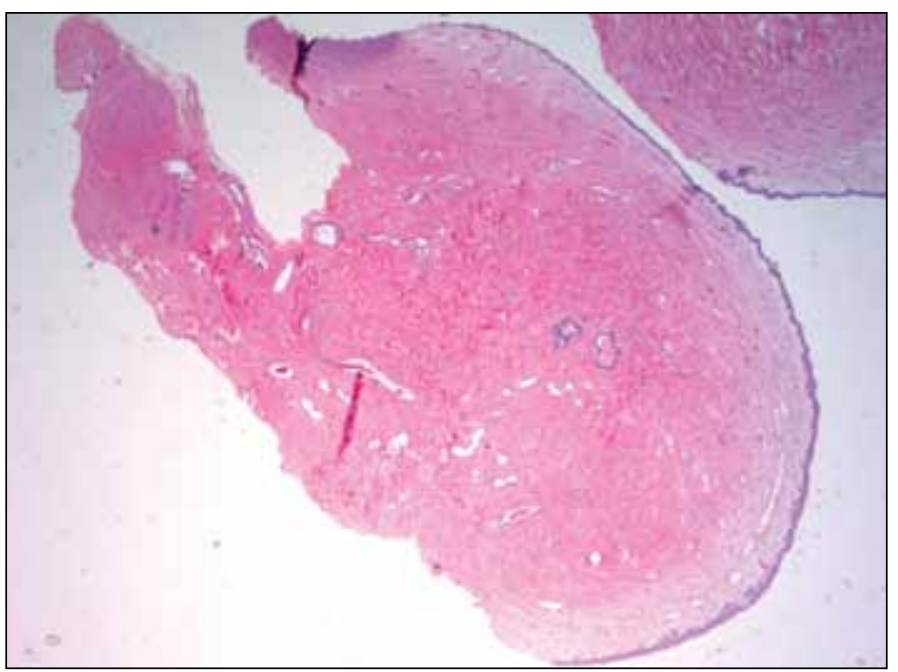

Fig. 2. Microphotograph $(\times 12.5$, Hematoxylin and eosin staining) of the fibroepithelial polyp demonstrating fibrovascular nature.

\section{Case 3}

A healthy 4-year-old boy presented with a febrile UTI. His mother described intermittency to his stream and pneumaturia. Ultrasound revealed a papillary $1 \times 1 \times 1.5-\mathrm{cm}$ mass at his bladder neck, whose vascular nature was confirmed on computed tomography scan (Fig. 4). Subsequent cystoscopy revealed a sessile, solid, non-papillary mass arising from his bladder neck/proximal prostate.

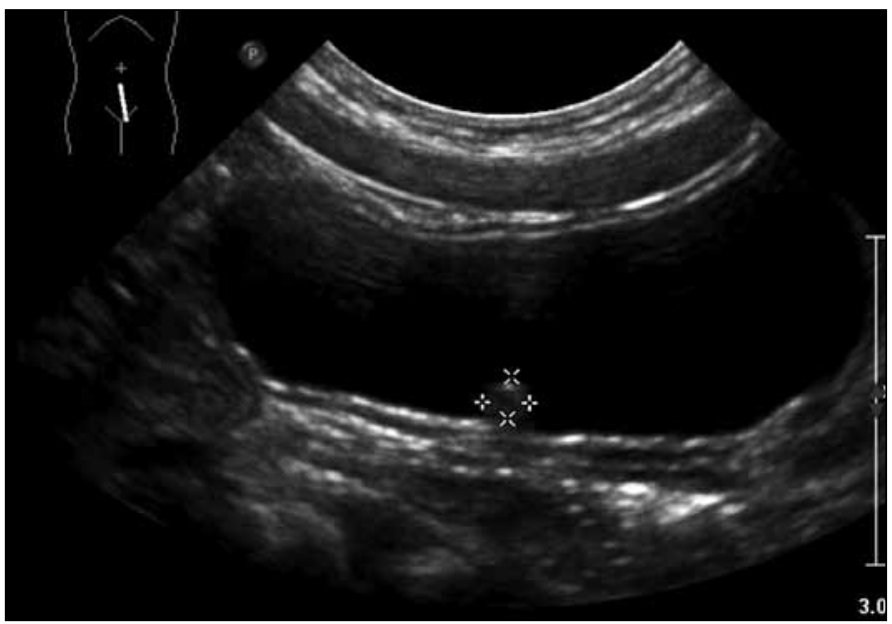

Fig. 3. Postnatal ultrasound (secondary to prenatal hydronephrosis) demonstrating asymptomatic $0.3 \times 0.3-\mathrm{cm}$ polypoid mass. 


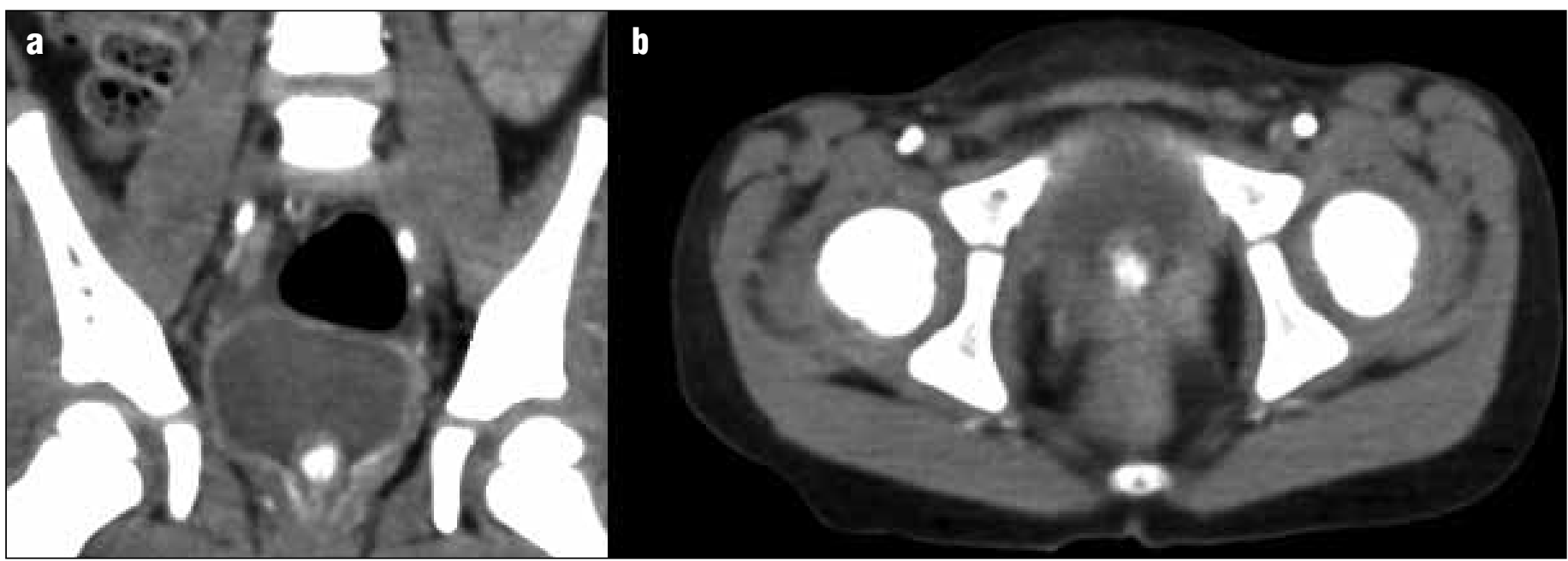

Fig. 4. A computed tomography scan of hemangioma demonstrating $1.3 \times 1 \times 1-\mathrm{cm}$ hypervascular mass at bladder neck.

Transurethral resection specimen described a hemangioma, and due to the technical difficulties of a complete transurethral resection, an open resection was performed (Fig. 5). Pathology revealed a benign appearing hemangioma (Fig. 6).

\section{Case 4 and Case 5}

Two teenage males ( 14 and 16 years old) presented with long-standing gross hematuria (6 and 12 months duration, respectively). Both were previously healthy, with no obvious environmental exposures. The 16-year-old performed small amounts of welding as a hobby and a career; the 14-yearold had a father who smoked in the house. Neither patient admitted to personal tobacco use. An ultrasound of both patients demonstrated tumours, which appeared to be typical papillary tumours (Fig. 7).

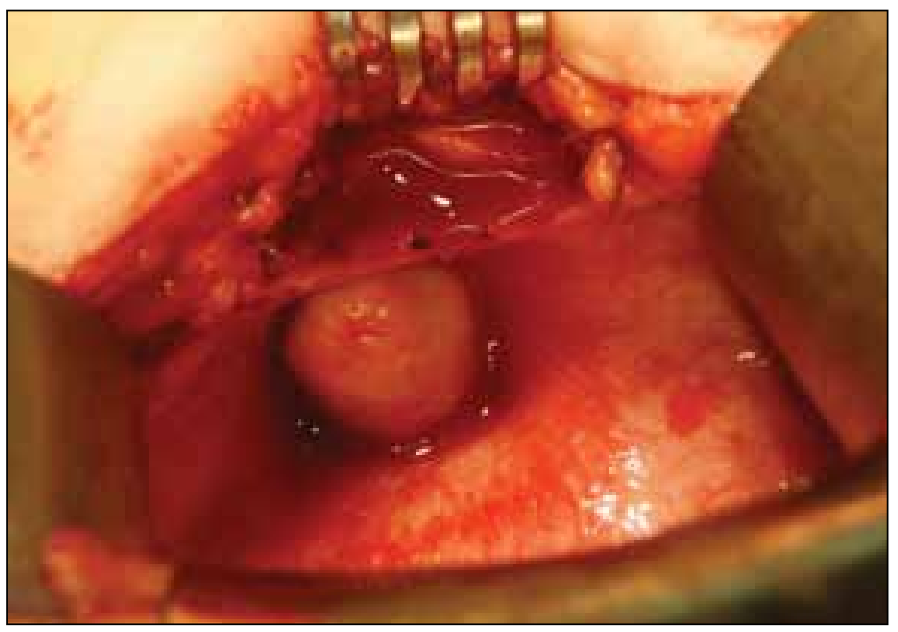

Fig. 5. Intra-operative photograph demonstrating the tumour occupying most of bladder neck.
Both patients underwent uncomplicated transurethral resection and microscopy was consistent with PUNLMP (Fig. 8). Three-month postoperative ultrasounds were negative. As per the recommendation of an adult uro-oncologist, surveillance cystoscopy was planned after 1 year.

\section{Discussion}

The presentation of these lesions can be quite similar to extremely common pediatric complaints secondary to benign etiologies and outcomes. However, subtle, important clues on history can distinguish these cases from more benign etiologies. Intermittent and interrupted voiding symptoms are very rare in pediatrics, and should alert the practitioner to possible abnormal pathophysiology. The association with UTI should raise the possibility of incomplete emptying. Although gross hematuria is common in pediatrics, the

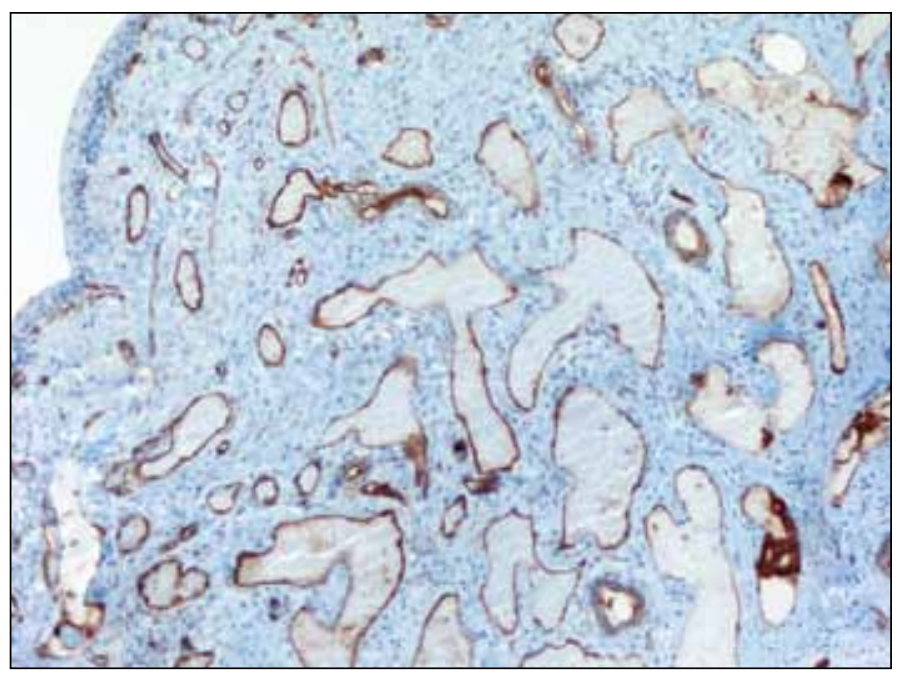

Fig. 6. Microscopy ( $\times 100$, anti-CD34 ABC immunostaining) of hemangioma specimen stained with $\mathrm{CD}-34$. 


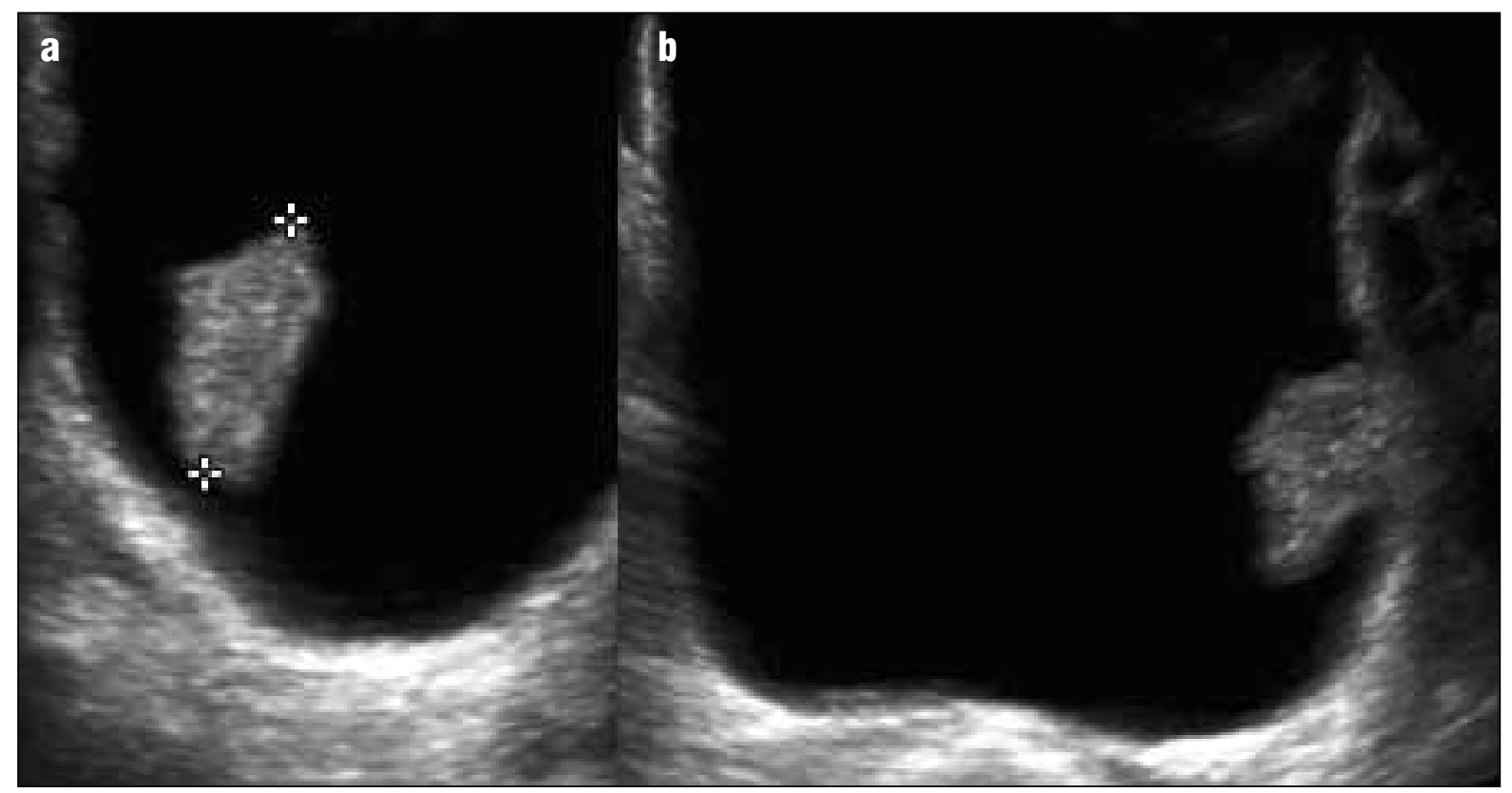

Fig. 7. Ultrasound of papillary urothelial neoplasms of low malignant potential in 2 separate teenagers who presented with gross hematuria which persisted for several months.

prolonged duration in the absence of a UTI or nephrologic pathology makes an anatomic lesion more likely. However, rhabdomyosarcoma and diethylstilbestrol (DES) exposure during pregnancy must be ruled out.

Urethral polyps are an anomaly, and the etiology is unknown. The incidence has increased in the past 20 years, possibly owing to better diagnostic techniques and more rigorous investigation into lower urinary tract symptoms. Fibroepithelial polyps have a variety of proposed etiologies including: congenital development, infectious, inflammatory, or secondary to an obstructive process. ${ }^{5}$ The most common etiology in the pediatric population is thought to be congenital, and occurs primarily in the posterior urethra or bladder. ${ }^{6}$ The most common presenting symptoms include gross hematuria, urinary retention, straining to void, polyuria and lower abdominal pain. ${ }^{5}$

Hemangiomas of the bladder have been frequently reported, and although mostly benign, ${ }^{7}$ significant bleeding has been reported. ${ }^{8}$

Papillomas are very uncommon, accounting for only $2 \%$ of adult transitional cell carcinomas. ${ }^{1,2}$ A series of 26 patients included only 1 child (8 years old) and most did not have recurrence. However, a 49 -year-old male, on immunosuppressive therapy for a renal transplant, developed a high-grade invasive tumour during follow-up. ${ }^{9}$ PUNLMPs accounted for most bladder malignancies in a recent SEER review. ${ }^{1}$ Although most pediatric tumours may be considered congenital, this is controversial. Shukla's review expresses concern about an increasing incidence, potentially secondary to environmental toxins, ${ }^{4}$ while other reports have included common adult carcinogens, such as schistosomia- sis, industrial exposure ${ }^{10,11}$ and radiation. ${ }^{12}$ Smoking is felt to be much less of a factor in patients $<20$ years old. ${ }^{3}$

Ultrasound is an extremely valuable tool in pediatric urology, and its sensitivity in these cases seems very good, and has also been reported in several small series. ${ }^{13,14}$ Although we do not have a proper denominator, all of these tumours

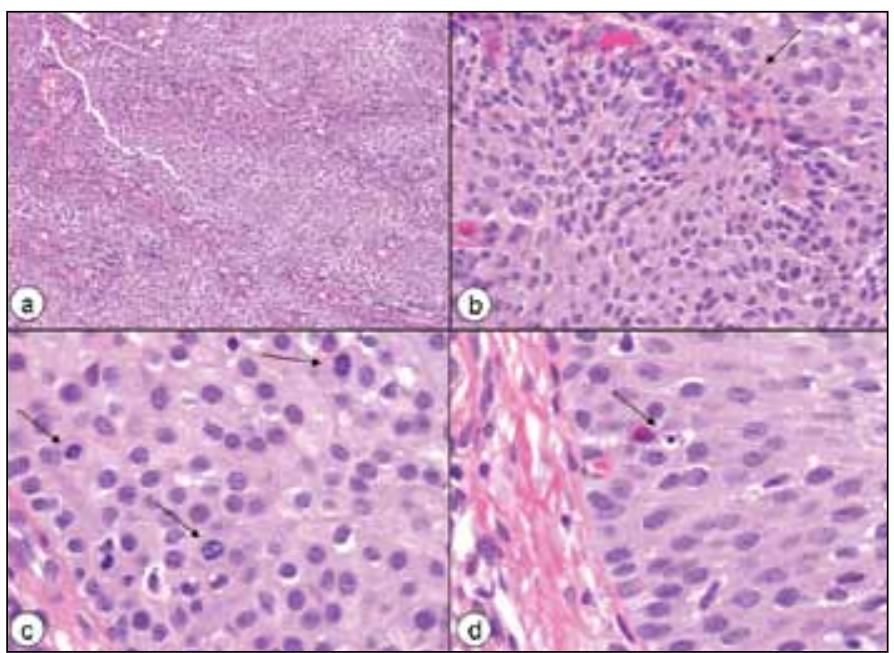

Fig. 8. Microphotograph of the papillary urothelial neoplasm of low malignant potential showing discrete and non-fused papillae, which are lined by multilayered urothelium with minimal to absent cytologic atypia. There is an increase of cell density as compared to normal urothelium (A). However, the polarity, particularly of the basal layer ( $B$, arrow), is preserved with palisading and an impression of predominant order is given. Obviously, there is also minimal variation in architectural and nuclear features and the nuclei are enlarged as compared to normal urothelial cells (B). Mitoses are rare, but are occasionally seen, although are not atypical (C, arrows). An apoptotic body is also seen (D, arrow) (Hematoxylin-eosin staining, a: $\times 100, b: \times 400, \mathrm{c}: \times 630, \mathrm{~d}: \times 630$ ). 
were accurately identified, when compared to cystoscopic and pathologic findings. We plan on following the 3 young children primarily with ultrasound, but will do one surveillance cystoscopy 1 -year post-resection for the 2 teenagers with the PUNLMP. Although potentially lacking the sensitivity of cystoscopy, ultrasound is acceptable and desirable in the pediatric population, as described by other authors. ${ }^{15}$ Many series report recurrences, and the authors of these reports suggest long-term follow-up. ${ }^{16}$ Therefore, we believe these patients merit follow-up, with the benign tumours (papilloma and PUNLMP) followed with at least one postoperative cystoscopy and annual surveillance with clinic visit, urine cytology and an ultrasound. The fibrovascular polyps and hemangiomas are more likely due to a congenital malformation and likely can be followed clinically and with ultrasound.

\section{Conclusion}

We present 4 different bladder tumours in 5 patients under 18 years old to demonstrate the potential variability in presentation. This cohort includes the youngest patient with a urological tumour and the first discovered by prenatal ultrasound. Given the environmental nature of adult tumours and the apparent increasing incidence in the pediatric population, we feel that a formal reporting program would improve monitoring and improve our knowledge base.

Competing interests: None declared.

This paper has been peer-reviewed.

\section{References}

1. Javadpour N, Mostofi FK. Primary epithelial tumors of the bladder in the first two decades of life. J Urol 1969:101:706-10

2. Kutarski PW, Padwell A. Transitional cell carcinoma of the bladder in young adults. Br J Urol 1993;72(5 Pt 2):749-55. http://dx.doi.org/10.1111/i.1464-410X.1993.tb16261.x

3. Paner GP, Zehnder $P$, Amin AM, et al. Urothelial neoplasms of the urinary bladder occurring in young adult and pediatric patients: a comprehensive review of literature with implications for patient management. Adv Anat Pathol 2011;18:79-89. http://dx.doi.org/10.1097/PAP.0b013e318204c0cf

4. Alanee $S$, Shukla AR. Bladder malignancies in children aged $<18$ years: results from the Surveillance, Epidemiology and End Results database. BJU Int 2010;106:557-60. http://dx.doi.org/10.1111/i.1464-410X.2009.09093.x

5. Demircan $M$, Ceran C, Karaman A, et al. Urethral polyps in children: a review of the literature and report of two cases. Int J Urol 2006;13:841-3. http://dx.doi.org/10.1111/i.1442-2042.2006.01420.x

6. Isaac J, Snow B, Lowichik A. Fibroepithelial polyp of the prostatic urethra in an adolescent. J Pediatr Surg 2006;41:e29-31. http://dx.doi.org/10.1016/i.pedsurg.2006.09.066

7. Tavora F, Montgomery E, Epstein Jl. A series of vascular tumors and tumorlike lesions of the bladder. Am J Surg Pathol 2008;32:1213-9. http://dx.doi.org/10.1097/PAS.0b013e31816293c5

8. Numanoglu KV, Tatti D. A rare cause of hemorrhagic shock in children: bladder hemangioma. J Pediatr Surg 2008;43:el-3. http://dx.doi.org/10.1016/i.jpedsurg.2008.02.057

9. McKenney JK, Amin MB, Young RH. Urothelial (transitional cell) papilloma of the urinary bladder: a dinicopathologic study of 26 cases. Modern Pathology 2003; 16:623-9. http://dx.doi.org/10.1097/01.MP.0000073973.74228.1E

10. Cherrie RJ, Lindner A, deKernion JB. Transitional cell carcinoma of bladder in first four decades of life. Urology 1982 Dec;20(6):582-4. http://dx.doi.org/10.1016/0090-4295(82)90303-X

11. Benton B, Henderson BE. Environmental exposure and bladder cancer in young males. J Natl Cancer Inst 1973;51:269-70.

12. Mincov N, Kyourktchieva S, Christozova I. Epithelial tumors of the urinary tract in children. Ann Urol (Paris) 1992;26:313-7.

13. Avolio L, Romano P, Parigi GB, et al. Bladder papilloma in a 12-year-old girl. J Pediatr Urol 2005;1:311-3. http://dx.doi.org/10.1016/i.jpurol.2005.01.006

14. Dennery MP, Rushton HG, Belman AB. Sonography for the detection and follow-up of primary nonsarcomatous bladder tumors in children. Urology 2002;59:119-21; discussion 121-2.

15. Hoenig DM, McRae $S$, Chen SC, et al. Transitional cell carcinoma of the bladder in the pediatric patient. J Urol 1996;156:203-5. http://dx.doi.org/10.1016/S0022-5347(01)66000-2

16. Paduano L, Chiella E. Primary epithelial tumors of the bladder in children. J Urol 1988;139:794-5.

Correspondence: Dr. Peter D. Metcalfe, Pediatric Urologist, Stollery Children's Hospital, Assistant Professor, University of Alberta, 2C3.79 Walter Mackenzie Centre, 8440-112 Street, Edmonton, AB T6G 2B7; pmetcalf@ualberta.ca 